\title{
Survey of different Clustering Algorithms used to Increase the Lifetime of Wireless Sensor Networks
}

\author{
Satyajeet R. Shinge \\ Department of Computer Engg. \\ Pimpri Chinchwad College of Engg. Savitribai \\ Phule Pune University, India.
}

\author{
S. S. Sambare \\ Associate Professor, \\ Department of Computer Engg. \\ Pimpri Chinchwad College of Engg. Savitribai \\ Phule Pune University, India.
}

\begin{abstract}
Wireless Sensor Networks (WSNs) have gained increasing attention from both the research community and actual user. WSN consists of a large number of sensor nodes which are deployed over an area to perform local computations based on information gathered from surroundings and send this information to the main location. Each sensor node is equipped with a limited battery- power. The energy, the storage capacity and communication capability of sensor nodes are very limited. It is almost difficult to change or replace the battery. One of the fundamental design challenges in designing a Wireless Sensor Network is to maximize the network lifetime. To overcome this challenge, different methods were developed in last few years.

The main objective in designing of WSN is to maximize the lifetime of WSN through minimizing the energy. There are different energy-efficient Clustering algorithms which prolongs the efficiency of WSN. Low-Energy Adaptive Clustering Hierarchy (LEACH) Protocol, Particle swarm optimization (PSO), Artificial Bee Colony (ABC) algorithm are different clustering mechanisms used to improve the efficiency of WSN. This paper discusses these three algorithms and compares this three with each other.
\end{abstract}

\section{Keywords}

WSN, LEACH, Lifetime, PSO, ABC, Cluster-Head

\section{INTRODUCTION}

Wireless Sensor Network (WSN) consists of large numbers of sensor nodes which are deployed in harsh and inaccessible terrains. WSN is very popular for monitoring the remote environments ${ }^{[6]}$.These sensor nodes monitors the parameters like temperature, humidity, vibration, etc. After monitoring these parameters it processes these data and transmits it to the base station or main location. Data is transfer to the main station in multi-hop routing. That means data monitored by sensor node is transmitted along node by node and finally reach to the base station. Limited memory and battery power are main constraints in WSN. So, it transmits data to Cluster-Head $(\mathrm{CH})^{[2]}$ of the cluster instead of direct transmission to base station. Transmitting data to $\mathrm{CH}$ is more energy efficient than direct transmission.

The main idea of clustering is to use Cluster-Head to collect data from other nodes and send it to base station. It reduces the energy dissipation of other nodes. LEACH is a well known protocol in Wireless Sensor Network. It is useful to increase the network Lifetime. The LEACH protocol is developed by Dr. Wendi Rabnir Heinzelman in $2000^{[2]}$. Clustering and Clustering-Heads in the LEACH are selected randomly. LEACH divides the sensor nodes in to different clusters and selects the $\mathrm{CH}$ from the clusters. If sensor nodes have data to send, they send it to the Cluster-Head. $\mathrm{CH}$ collects data from other nodes, processes those data and transmits data to other
CH's or base station. Also Particle Swam Optimization (PSO) and Artificial Bee Colony (ABC) algorithm is two swarmbased artificial intelligence algorithms, used for the clustering mechanism. . PSO algorithm is based on the migration behaviour of flock birds for the process of searching food ${ }^{[4]}$ Artificial bee colony algorithm is inspired by behavior of honey bees.

\section{LITERATURE SURVEY \\ 2.1 LEACH Protocol}

LEACH protocol is used for the cluster formation of the sensor network. This protocol forms the cluster of the nodes by using distributed algorithm and select Cluster Head for each cluster by some probability. Once the cluster is formed other nodes select the $\mathrm{CH}$ to join cluster. The nodes in the cluster access the $\mathrm{CH}$ according to Time Division Multiple Access (TDMA) ${ }^{[2]}$. The Cluster-Heads of different cluster communicate in a Code Division Multiple Access (CDMA) ${ }^{[2]}$. When nodes have data to send to the base station, they send their data to the ClusterHead. $\mathrm{CH}$ collects data from nodes and sends it to the base station. After some time, the new $\mathrm{CH}$ is formed by repeating the cluster formation algorithm.

\subsubsection{Cluster Formation Process of LEACH}

There are two phases in cluster formation using LEACH Protocol ${ }^{[1]}$.

\section{$>$ Set-Up Phase}

In Set-Up Phase there are two phases:

(1)Advertisement Phase

(2) Cluster Set-Up Phase

In the Advertisement Phase, the $\mathrm{CH}$ send the advertisement packet to their nearby nodes to inform that they become Cluster-Head. In the Cluster Set-Up phase, non-CH nodes send "join-packets", to $\mathrm{CH}$ to inform that they become the member of the cluster.

\section{$>$ Steady Phase}

The actual transmission of data begins in this phase. When TDMA slot is allocated to any node, they send their data to their $\mathrm{CH}$. If TDMA slot is not allocated to non- $\mathrm{CH}$ node, their transceiver can be turned off. After receiving all the data, $\mathrm{CH}$ aggregates the data and sends to the final Location.

Although, LEACH Protocol gives good result, it suffers from many drawbacks ${ }^{[1]:}$

$>$ It is applicable to only small area.

$>\mathrm{CH}$ 's are non-uniformly distributed and $\mathrm{CH}$ can be located at the edge of cluster. 
$>$ Cluster-Head selection is selected randomly, that does not take into account energy consumption.

\subsection{Particle Swarm Optimization}

Particle Swam Optimization is simple, effective and efficient optimization algorithm. PSO is used to achieve the minimum intra-cluster distance. PSO algorithm is based on the migration behaviour of flock birds for the process of searching food. For the process of food searching, each bird can find food with the social collaboration with neighbouring birds. A bird who has found the food can guide the other birds search for the location of food. Once these birds find food, they can guide other birds to find the location of food, which increases the possibility of bird flock finding food[3].

The operation of this protocol is based on a centralized control algorithm that is implemented at the base station, which is a node with a large amount of energy supply. At the starting of cluster formation step, all nodes send their current energy status and their location to the base station. Based on this information, nodes having sufficient energy are selected as a cluster-Head. The PSO algorithm applied during the cluster setup phase is as follows [3]:

1. Initialize an $\mathrm{S}$ particle that contains randomly selected Cluster-Heads.

2. Evaluate the cost function of each particle:

i. For each node ni, $i=1,2, \ldots, N$

-Calculate distance d(ni,chp,k) between node ni and all cluster heads chp,k.

-Assign node ni to cluster head chp,k where:

$$
d\left(n i, c h_{p, k}\right)=\min \forall k=1,2, \ldots, k\left\{d\left(n i, c h_{p, k}\right)\right\}
$$

ii. Calculate the cost function using equations:

$$
\cos t=\beta \times f 1+(1-\beta) \times f 2
$$

Where, $\mathrm{f} 1$ is the maximum average Euclidean distance of nodes to their associated cluster heads and $\mathrm{f} 2$ is the ratio of total initial energy of all nodes in the network with the total current energy of the cluster heads candidates in the current round and is given by

$$
\begin{gathered}
f 1=\max k=1,2, \ldots, k\left\{\sum_{\forall n i \in C p, k} d(n i, c h p, k) /|C p, k|\right\} \\
f 2=\sum_{i=1}^{N} E\left(n_{i}\right) / \sum_{k=1}^{K} E\left(c h_{p, k}\right)
\end{gathered}
$$

3. Find the personal and global best for each particle.

4. Update the particle's velocity and position using

$$
\begin{aligned}
& v_{i d}(t)=w \times v_{i d}(t-1)+c 1 \varphi 1\left(p_{i d}-x_{i d}(t-1)\right)+c 2 \varphi 2\left(p_{s d}-x_{i d}(t-1)\right) \\
& x_{i d}(t)=x_{i d}(t-1)+v_{i d}(t)
\end{aligned}
$$

Here, $v$ and $x$ are the particle velocity and particle position respectively, $t$ is Time, $c 1, c 2$ are Learning factors, $\phi 1, \phi 2$ are Random numbers between 0 and 1, pid is Particle's best position, pgd is Global best position and $\mathrm{w}$ is Inertia weight.

5. Limit the change in the particle's position value.

6. Map the new updated position with the closest $(\mathrm{x}, \mathrm{y})$ Coordinates.
7. Repeat steps 2 to 6 until the maximum number of iterations is reached.

\subsection{Artificial Bee Colony Algorithm}

Artificial Bee Colony (ABC) is bio-inspired algorithm used to improve the efficiency in Wireless Sensor Network. . c It is energy efficient clustering mechanism. $\mathrm{ABC}$ has been successfully used in clustering techniques. $\mathrm{ABC}$ algorithm is inspired by behavior of honey bees [5].

In $\mathrm{ABC}$, search procedure is carried out by three groups of bees as employee, onlooker and scout bees. Employed bees search food sources within the neighborhood of the initial food sources in their memory and they share new food sources information with onlooker bees. These onlooker bees then search a new food source according to the received Information, i.e., finding out a new one from the neighborhood of existing food sources. Scout bees search a new food source randomly when an employed bee abandons a food source. At the beginning, $\mathrm{ABC}$ algorithm generates a large number of food sources randomly. A food source represents a possible solution. The employed bees produce a modification on the position of the food source in their memory ${ }^{[5]}$.

The quality of a food source, i.e., the amount of nectar, is evaluated by its fitness value. Then, the algorithm calculates all the food sources' selection probabilities by their fitness values. The onlooker bees select the food source with the largest probability value and produce a modification on the position of the food source. The fitness value of the new food source is calculated and compared with the fitness value of the old one. Then, the food source with the larger fitness value is recorded as the temporarily best solution. The best food source is iteratively updated for certain iterations. The pseudo-code of $\mathrm{ABC}$ algorithm as follows:

- Generate initial population $\mathrm{Xi}, \mathrm{i}=1 \ldots \mathrm{SN}$

- Evaluate the population

- $\quad$ Set cycle to 1

- Repeat

- $\quad$ FOR each employed bee

- $\quad$ Produce new solutions vi by using

$$
v i_{j}=x i_{j}+\theta_{i j}\left(x_{i j}-x_{k j}\right)
$$

- Calculate the fitness

- Apply the greedy selection process

- $\quad$ FOR each onlooker bee

- $\quad$ Choose a solution xi depending on pi

- $\quad$ Produce new solutions vi

$$
p_{i}=\frac{f_{i t}{ }_{i}}{\sum_{n-1}^{S N} f i t_{n}}
$$

- Calculate the fitness

- Apply the greedy selection process

- If there is an deserted solution then

- Replace this solution with a new solution produced by a scout using 


$$
x_{i}^{j}=x_{\min }^{j}+\operatorname{rand}(0,1)\left(x_{\max }^{j}-x_{\min }^{j}\right)
$$

- Store the best solution achieved till

- $\quad$ cycle $=$ cycle +1

- $\quad$ Until cycle $=\mathrm{MCN}$

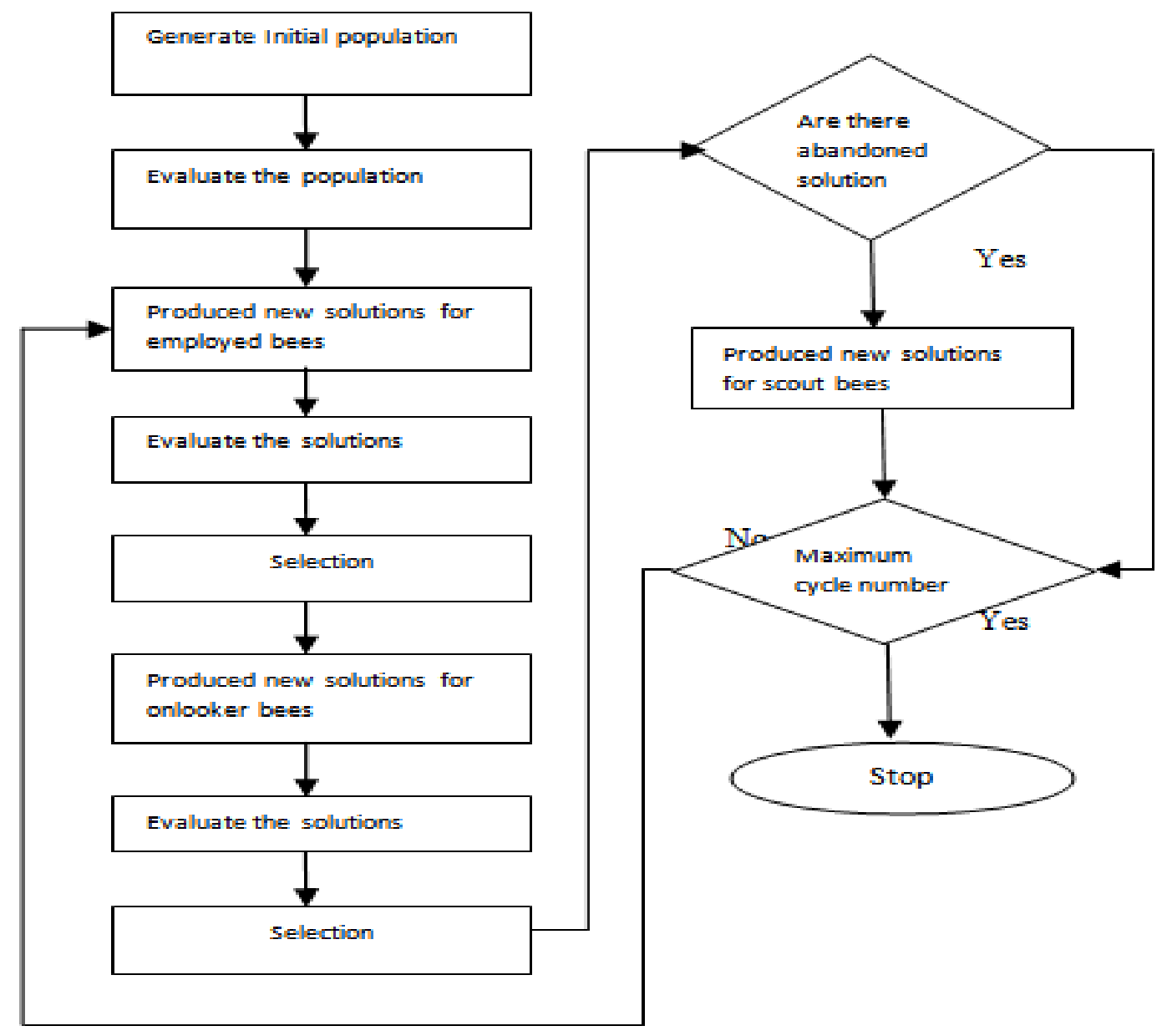

Figure 1. Flow chart for Artificial Bee Colony Algorithm

\section{COMPARATIVE ANALYSIS}

Table 1. Comparison between LEACH, PSO and ABC

\begin{tabular}{|c|c|c|c|c|c|c|}
\hline & $\begin{array}{l}\text { Selection of cluster } \\
\text { Head }\end{array}$ & $\begin{array}{l}\text { Location of cluster } \\
\text { Head }\end{array}$ & $\begin{array}{l}\text { Distance between } \\
\text { CH and non-CH } \\
\text { nodes }\end{array}$ & No. of clusters & $\begin{array}{l}\text { Intra } \\
\text { cluster } \\
\text { distance }\end{array}$ & $\begin{array}{l}\text { Number of nodes } \\
\text { alive }\end{array}$ \\
\hline LEACH & $\begin{array}{l}\mathrm{CH} \text { selection is } \\
\text { randomly. It is not } \\
\text { depend upon energy } \\
\text { consumption }\end{array}$ & $\begin{array}{l}\mathrm{CH} \text { are non- } \\
\text { uniformly distributed } \\
\text { i.e. at the edge of } \\
\text { cluster }\end{array}$ & $\begin{array}{l}\text { Distance between } \\
\mathrm{CH} \text { and non-CH } \\
\text { nodes is not } \\
\text { minimized }\end{array}$ & $\begin{array}{l}\text { Does not ensures } \\
\text { the expected } \\
\text { number of Clusters }\end{array}$ & $\begin{array}{l}\text { Does not } \\
\text { gives } \\
\text { guarantee } \\
\text { for } \\
\text { minimum } \\
\text { intra-cluster } \\
\text { distance }\end{array}$ & $15000-25000$ \\
\hline
\end{tabular}




\begin{tabular}{|l|l|l|l|l|l|l|}
\hline PSO & $\begin{array}{l}\mathrm{CH} \text { selection is based on } \\
\text { energy consumed by the } \\
\text { sensor nodes. }\end{array}$ & $\begin{array}{l}\mathrm{CH} \text { are optimally } \\
\text { distributed across the } \\
\text { network }\end{array}$ & $\begin{array}{l}\text { Distance between } \\
\mathrm{CH} \text { and non-CH } \\
\text { nodes is minimized } \\
\text { expected number } \\
\text { of clusters }\end{array}$ & $\begin{array}{l}\text { Achieve } \\
\text { minimum } \\
\text { intra-cluster } \\
\text { distance. }\end{array}$ & $30000-40000$ \\
\hline ABC & $\begin{array}{l}\mathrm{CH} \text { selection is based on } \\
\text { the fitness value } \\
\text { calculated by each node. }\end{array}$ & $\begin{array}{l}\mathrm{CH} \text { are optimally } \\
\text { distributed }\end{array}$ & $\begin{array}{l}\text { Minimum distance } \\
\text { between CH and } \\
\text { non-CH nodes }\end{array}$ & $\begin{array}{l}\text { Ensures } \\
\text { expected number } \\
\text { of Clusters }\end{array}$ & $\begin{array}{l}\text { intra-cluster } \\
\text { distance } \\
\text { minimum }\end{array}$ & $\begin{array}{l}\text { More than 40000 } \\
\text { is }\end{array}$ \\
\hline
\end{tabular}

\section{CONCLUSION}

From above comparison it is conclude that LEACH provides significant energy savings and prolonged network lifetime but does not guarantee that the desired number of cluster heads is selected and cluster heads are not evenly positioned across the network. Also it does not give minimum distance between $\mathrm{CH}$ and non- $\mathrm{CH}$ nodes.

Whereas, in PSO and ABC algorithm Cluster-Heads are optimally distributed. These two algorithms give minimum distance between $\mathrm{CH}$ and non- $\mathrm{CH}$ nodes. Artificial Bee Colony gives more alive nodes as compare to $\mathrm{LEACH}$ and PSO algorithm. So, ABC algorithm is used to increase the Lifetime of WSN.

\section{REFERENCES}

[1] Nikhil Marriwala, Priyanka Rathee, "An Approach to Increase the Wireless Sensor Network Lifetime", 978-14673-4805-8/12/\$31.00_c 2012 IEEE .

[2] Hu Junping, Jin Yuhui, Dou Liang, "A Time-based Cluster-Head Selection Algorithm for LEACH", 978-14244-2703-1/08/\$25.00 (C2008 IEEE.

[3] Nikitha Kukunuru, Babu Rao Thella, Rajya Lakshmi Davuluri, "Sensor Deployment Using Particle Swarm Optimization", International Journal of Engineering Science and Technology Vol. 2(10), 2010, 5395-5401.
[4] N. M. Abdul Latiff, C. C. Tsimenidis, B. S. Sharif, "Energy-Aware Clustering For Wireless Sensor Networks Using Particle Swarm Optimization", The 18th Annual IEEE International Symposium on Personal, Indoor and Mobile Radio Communications.

[5] Dervis Karaboga, Selcuk Okdem,Celal Ozturk, "Cluster based wireless sensor network routing using artificial bee colony algorithm", Published online: 24 April 2012 Springer Science+Business Media, LLC 2012.

[6] Mrs. Rakhi Khedikar, Dr.Avichal Kapur, Dr. M. D. Chawhan, "Energy Efficient Wireless Sensor Network", 2014 International Conference on Electronic Systems, Signal Processing and Computing Technologies.

[7] Satyesh Sharan Singh, Mukesh Kumar, Rohini Saxena, Priya, "Application of Particle Swarm Optimization for Energy Efficient Wireless Sensor Network", International Journal of Engineering Science \& Advanced Technology.

[8] Suraj B. Borge, Mayura U. Kinikar, "Artificial Bee Colony (ABC) algorithm to improve Efficiency in Wireless Sensor Networks (WSNs)". 